\title{
LE VOCABULAIRE DU TOURISME, UN LABORATOIRE EXPÉRIMENTAL DES PROCÉDÉS NÉOLOGIQUES
}

\author{
GIOVANNI TALLARICO
}

\begin{abstract}
The vocabulary of tourism, an experimental lab for word formation

The vocabulary of tourism has not been studied in depth until recently. In this paper we try to fill this gap, by focussing on French neologisms attested in this field. Our research, based on Timestamped JSI web corpus (2014-2019), shows that word formation largely stems from binomial appositions, thus following the model of terminology. A comparatively small number of words consist of translations from English, since French tourism is deeply rooted in national culture.
\end{abstract}

Keywords : Neology, tourism, word formation, anglicisms, regional variation

Mots clés : Néologie, tourisme, procédés de formation, anglicismes, variation diatopique

\section{Introduction}

Fait économique majeur à l'échelle globale, phénomène polyvalent ${ }^{1}$, objet d'un débat culturel et médiatique de plus en plus vif (et souvent critique) ${ }^{2}$, le tourisme est une composante fondatrice et incontournable de l'époque actuelle ${ }^{3}$. Le rapport privilégié que le tourisme entretient avec les innovations technologiques et son rôle de catalyseur des évolutions sociétales en font un terrain d'enquête stimulant pour toutes les sciences humaines.

En ce qui concerne les aspects linguistiques, le vocabulaire du tourisme connaît une expansion fulgurante ${ }^{4}$. Pourtant, il est assez peu traité, aussi bien en terminologie officielle $^{5}$ qu'en sciences du langage $^{6}$, où de toute façon sont privilégiées les approches

Cf. Decroly et al. (2006); Kalfon (2009).

Cf. Paquot (2014); Christin (2014).

Cf. Angelillo (2015).

Cf. Tallarico, Humbley (2020).

5 La base de données FranceTerme (www.culture.fr/franceterme) ne répertorie à ce jour que 35 termes du domaine "tourisme ", mais ce nombre assez exigu peut aussi s'expliquer par un faible taux de pénétration des anglicismes dans ce secteur. $C f$. aussi Renner (2012).

6 Nigro (2006:75) observe quel'absence d'études systématiques du langage du tourisme est due sans doute à sa complexité et à la richesse de ses composantes multidisciplinaires. $C f$. aussi Piccato $(2014,2015)$. 
discursives et liées aux genres textuels (notamment les guides touristiques) ${ }^{7}$. Les raisons en sont multiples : le domaine est par sa nature interdisciplinaire et sa " disciplinarisation » est assez récente ${ }^{8}$. Preuve en est un échantillon de comptes rendus de thèses de doctorat soutenues dans ce domaine, publiés par la revue Mondes du tourisme ${ }^{9}$. Visiblement, les travaux portant sur le tourisme ont été préparés dans des cadres disciplinaires très disparates : anthropologie, géographie, histoire, sociologie, management, communications, lettres, urbanisme, ethnologie, jusqu’à des dénominations plus spécifiques telles «études touristiques» (Lausanne) ou " sciences du tourisme » (Perpignan). Comme l'observe Paquot, « les diverses disciplines universitaires se sont emparées différemment de cet objet d'étude qu'est devenu le tourisme » (2014:20).

Le but de cette contribution est d'étudier la néologie dans le domaine touristique, en nous concentrant surtout (mais non exclusivement) sur les acteurs principaux de ce phénomène socio-culturel, les touristes eux-mêmes, et sur les pratiques dont ils sont protagonistes, les nouvelles formes de tourisme.

\section{Méthodologie}

Afin d'analyser les néologismes dans ce secteur, nous avons exploité le corpus Timestamped JSI web (2014-2019) ${ }^{10}$, disponible sur le système de gestion Sketch Engine ${ }^{11}$. En ce qui concerne le français, ce vaste corpus comporte environ 5 milliards de mots et regroupe des textes issus de la presse et du web francophones. À partir d'une recherche menée sur les formes contenant ${ }^{*}$ tourism ${ }^{*}$ et ${ }^{*}$ tourist ${ }^{* 12}$, la série regroupant ces deux bases a été successivement importée dans la plateforme Néoveille-PHC ${ }^{13}$. Les contextes tirés de JSI ont été rendus directement disponibles dans Néoveille-PHC, où nous avons mené une catégorisation et une description des lexies.

Le nombre de lexies, environ mille à ce stade, a été par la suite réduit manuellement en éliminant le bruit, qui consistait pour la plupart en entités nommées. Après avoir exclu les lexies clairement non néologiques (comme tourisme, touriste, touristique), nous avons également éliminé des formes non pertinentes pour notre champ d'étude, telles que retourisme (hapax lié au domaine politique), construit par suffixation à partir de retour, ou encore super-tourisme ${ }^{14}$, car l'examen des contextes montre qu'il s'agit d'un terme lié au domaine des sports automobiles. La liste de lexies néologiques issues

\footnotetext{
Cf. Margarito (2000) ; Baider, Burger, Goutsos (2005) ; Gotti, Naci, Sala (2017).

8 Par exemple, Aquilina, Mahéo et Pugnière-Saavedra observent qu' « il est difficile de considérer le "tourisme" comme un objet de recherche structuré par un partage de catégories et de méthodologies ». D'ailleurs, « en France, au-delà du peu d'enquêtes de terrain et de chercheurs travaillant sur ce thème, les problématiques touristiques sont abordées depuis longtemps, et encore en partie aujourd'hui, de manière autonome par chaque champ disciplinaire» $(2018: 1)$.

9 https://journals.openedition.org/tourisme/, consulté le 25. 06. 2020.

Désormais : JSI.

www.sketchengine.eu, consulté le 25. 06. 2020.

12 L'astérisque indique que tout caractère peut se placer avant ou après la forme choisie comme base.

13 Ce sigle indique qu'il s'agit d'une extension de la plateforme Néoveille, qui a été développée dans le cadre du partenariat Hubert-Curien avec la République Tchèque (projet Barande) et la Pologne (projet Polonium).

14 Nous avons en revanche retenu la forme supertouristes, v. infra.
} 
des bases ${ }^{*}$ tourism ${ }^{*} /{ }^{*}$ tourist ${ }^{*}$ comporte environ 300 lexies (variantes orthographiques comprises).

Notre analyse concerne en priorité les procédés lexicogéniques à l'œuvre dans la série lexicale retenue. Cependant, nous signalerons aussi quelques relations de nature sémantique entre la lexie en question et d'autres lexies du corpus, au niveau de la synonymie, de l'hyperonymie, de l'hyponymie et co-hyponymie et de l'homonymie. L'étude des contextes, bien que limitée à quelques cas significatifs, permettra d'évaluer l'insertion en discours de la lexie concernée. Pour finir, nous allons aborder brièvement les rapports de certains néologismes avec les anglicismes correspondants et la question de la variation diatopique, telle qu'elle émerge de notre corpus.

\section{Analyse des résultats}

En ce qui concerne les parties du discours, dans le corpus étudié la néologie concerne en priorité les noms (124 lexies ${ }^{15}$ ), suivis des adjectifs (54 lexies). Le calcul des lexies néologiques est fastidieux car il doit impérativement tenir compte des nombreuses variantes orthographiques, par ex. cenotourisme, cno-tourisme ou encore oeno(-)tourisme, ultratouristique ou ultra-touristique ${ }^{16}$, ou encore les formes concurrentes agri(-) touristique / agro(-)touristique, où la variation concerne aussi bien la présence du trait d'union que la forme même du fractolexème : agri- ou agro-. Nous avons opéré une lemmatisation pour les cas des lexies soudées ou avec trait d'union, qui n'ont été comptabilisées qu'une fois, ce qui donne un total de 180 lexies « de travail ».

Nous avons pu repérer un seul adverbe de manière, touristiquement (187 occurrences : «Le centre-ville est bien mis en valeur touristiquement ${ }^{17}$ ), dont le statut néologique est discutable, non seulement par sa fréquence relativement élevée mais parce qu'il s'agit d'une formation parfaitement régulière, voire prévisible.

Dans le corpus est aussi attesté un verbe créé par conversion, tourister ( 3 occ.), qui peut être reformulé par l'expression avec verbe support «faire du tourisme » ( Peut-être partir tourister ${ }^{18}$ sur la banquise? $\left.»^{19}\right)$. Le sens de ce verbe semble proche de touristiquer, non attesté dans notre corpus mais figurant dans une publication d'un spécialiste qui crée volontiers des néologismes : "Le touriste n'est pas vacant, il doit impérativement “touristiquer”. C'est cela la finalité du tourisme » (Paquot 2012 : 19).

Passons maintenant à l'analyse des procédés néologiques attestés dans le corpus, en commençant par les noms. La composition se taille la part du lion, dans ses différentes sous-typologies. En toute première place, nous avons la composition " régulière $»^{20}$, qui

15 Pour le calcul des lexies, nous avons comptabilisé uniquement le mot-forme (ou lemme) : une lexie au singulier et son pluriel comptent donc pour une fois. Lorsque les formes au singulier et au pluriel figurent dans le corpus, dans cet article nous reprenons habituellement le singulier.

16 Sur la variation orthographique concernant les éléments de formation hyperboliques, $c f$. Jacquet-Pfau (2020).

17 www.centre-presse.fr, 19. 10. 2019. Dans les citations, sauf là où nous le précisons, les italiques sont de l'auteur de cet article.

18 En italique dans le texte.

19 www.agoravox.fr, 27. 08. 2018.

20 Nous n'étudions pas ici les composés non néologiques du type (service) animation-tourisme, (pôle) communication-tourisme, (point) info-tourisme, etc. 
concerne surtout les différentes formes de tourisme. Dans ce cas, le premier élément du composé indique le but, le moyen ou l'objet de la pratique touristique (le café, le cannabis, le cidre, le cinéma, la moto, la pêche, etc.) et désigne autant d'hyponymes de tourisme: café-tourisme, cannabo-tourisme, cidro(-)tourisme, ciné(-)tourisme, moto(-)tourisme, pescatourisme, photo(-)tourisme, récréotourisme, sexotourisme, shopping-tourisme ${ }^{21}$, spaciotourisme, sport-tourisme et vélo(-)tourisme.

Dans deux autres cas, le mot tourisme est en première position et un autre substantif s'y associe pour indiquer une typologie spécifique de tourisme : tourisme-charia ( Il a également demandé la promotion du 'tourisme-charia' qui respecterait les règles les plus strictes de l'islam » ${ }^{22}$ ) et tourisme-santé ( Nous avons une approche du thermalisme en termes de tourisme-santé $\left.»^{23}\right)$. On pourrait se demander s'il existe des différences sémantiques entre ces deux groupes de composés, mais le peu d'exemples pour cette deuxième catégorie n'autorisent pas de généralisations.

D’autres composés, en revanche, concernent des établissements touristiques «hybrides" (agrotourisme-camping) ou encore des phénomènes sociaux (tout-au-tourisme, tout-tourisme).

On peut également identifier une autre catégorie de substantifs composés, qui renvoient plutôt à des typologies de touristes, en fonction de leurs pratiques (agrotouristes-campeurs, moto-touristes, sexotouristes, techno-touristes ${ }^{24}$, vélo(-)touriste, volcano-touristes) ou de leurs caractéristiques distinctives (non-touriste, touriste-type).

Un autre groupe de lexies identifie des touristes qui ont une double appartenance catégorielle. Dans ce cas, décrit par Picone (1996 : 117) comme des «binomial appositions ", l'accent sémantique se place toujours sur le premier élément de la composition. Nous avons ainsi les formes touristes-alpinistes, touristes-astronautes, touristes-chasseurs, touristes-excursionnistes, touristes-routiers, touristes-supporters (composé hybride) et touristes-visiteurs, qui renvoient à des situations où, pour ainsi dire, les touristes sont aussi, et en même temps, des alpinistes/astronautes/chasseurs, etc. Dans les cas où touriste(s) est en deuxième position (coureurs-touristes, écoliers-touristes, pêcheurs-touristes, pilotes-touristes, piéton-touriste, voyageurs-touristes), il s'agit plutôt, nous semble-t-il, de coureurs/ écoliers/pêcheurs, etc. qui portent aussi une casquette de touriste ${ }^{25}$.

Pour finir, le composé chasse-touristes renvoie aux personnes qui gravitent « autour de

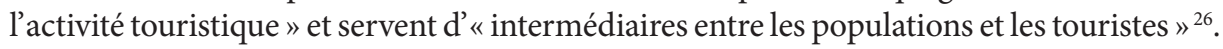

La composition hybride est également bien représentée ${ }^{27}$. Parmi les formes de tourisme, nous avons tout d'abord des lexies co-hyponymes où le premier élément (confixe issu du grec ou du latin) s'associe à la base indigène « tourisme " pour indiquer le but ou les

21 À la rigueur, il pourrait s'agir d'un composé hybride, mais shopping n'est sans doute plus perçu par les locuteurs comme un mot d'origine étrangère.

www.liberation.fr, 07. 04. 2017.

www.lamontagne.fr, 13. 04. 2019.

24 Il s'agit de touristes passionnés de musique techno et non pas de touristes « technologiques » (dans ce dernier cas, il s'agirait plutôt d'une composition hybride).

25 De même pour les hapax député-touriste et ministre-touriste, qui se bornent à décrire l'attitude d'un député canadien et d'une ministre française.

26 www.nigerdiaspora.net, 01. 07. 2016.

27 Pour une typologie fine des hybrides en français, $c f$. Kortas (2009). 
caractéristiques de la typologie touristique en question : aérotourisme, alphatourisme $e^{28}$, aqua(-)tourisme, astro(-)tourisme, avitourisme (tourisme ornithologique), cyclo(-)tourisme, égotourisme (lié à égoportrait, synonyme de selfie), ethno-tourisme, géo(-)tourisme ${ }^{29}$, géo-écotourisme, mycotourisme (tourisme lié aux champignons), narco(-)tourisme («Surfant sur le succès de Narcos, la ville de Medellín a vu se développer ces dernières années un juteux business du narcotourisme ${ }^{30}$ ), nécrotourisme ( Le nécrotourisme attire de plus en plus de visiteurs dans les cimetières bruxellois » ${ }^{31}$ ), néo-tourisme, œno(-)tourisme, oléo(-)tourisme (tourisme qui valorise l'huile d'olive) et thanatourisme («Un exemple de tourisme noir, ou thanatourisme, l'appellation de cette forme de visite des lieux associés à la mort ou à la catastrophe $»^{32}$ ). L'hapax vinotourisme est, quant à lui, un synonyme d'œno(-)tourisme.

D’autres composés hybrides, en revanche, dénotent une nouvelle « science du tourisme », la tourismologie $e^{33}$ ( De nouveaux vocables sont apparus, on parle désormais de tourismologie, une science propre qui permet toutes les remises en cause nécessaires ${ }^{34}$ ), l'aversion vers le tourisme (tourismophobie) ou les touristes (touristophobie).

Parmi les lexies hybrides qui se réfèrent à des catégories de touristes, nous avons repéré les formes suivantes : astrotouristes, cyclo(-)touriste ${ }^{35}$, électro-touristes (hapax désignant les touristes qui utilisent des voitures électriques), électrocyclotouristes, narcotouristes, nécrotouristes, néo-touristes, cno(-)touriste, pseudo-touriste(s) et touristonaute (consommateur de tourisme en ligne $\left.{ }^{36}\right)$. Les composés avec astro-, cyclo-, narco-, nécro-, néo- et œno- s'avèrent donc les plus productifs car ils se retrouvent dans les deux catégories, celle liée aux formes de tourisme et celle liée aux touristes eux-mêmes.

Il y a un doute d'attribution concernant les lexies suivantes : overtourisme, slow(-) tourisme, e(-)tourisme et e-touristes. En toute rigueur, il s'agirait de composés hybrides, qui associent une unité lexicale anglaise (ou un fractolexème, dans le cas de $e$-) à une française, mais on pourrait également les considérer comme de simples emprunts adaptés, surtout pour overtourisme et slow(-)tourisme.

La fractocomposition rend compte d'un nombre assez élevé de lexies, liées surtout aux formes de tourisme : agri(-)/agro(-)tourisme, bio(-)tourisme, brassitourisme (tourisme brassicole), can(n)atourisme (synonyme de cannabo-tourisme), choco(-)tourisme, éco(-)

28 «Le Laboratoire du tourisme expérimental (Latourex) propose des idées pour découvrir le monde de manière différente. Avec l'alphatourisme, il invite les visiteurs à parcourir une ville de $\mathrm{A}$ à $\mathrm{Z}$, de la première rue à la dernière selon l'ordre alphabétique " (www.courrier-picard.fr, 21. 08. 2016). Il s'agit d'une forme de tourisme "mettant en valeur l'originalité géographique d'un endroit, à savoir son environnement, sa culture, son esthétique, son patrimoine et le bien-être de ses habitants » (www.elwatan.com, 07. 10. 2017).

30 www.huffingtonpost.ca, 31. 01.2017.

31 www.bx1.be, 14.04. 2019.

32 www.20minutes.fr, 12. 06. 2019.

33 Le terme est aussi attesté dans le travail d'un spécialiste : Hoerner, J.-M. (2002) : Traité de tourismologie, pour une nouvelle science touristique, Perpignan : Presses universitaires de Perpignan. Dans une autre perspective, Paquot $(2014: 39)$ préfère parler de touristologie.

34 www.lexpressmada.com, 14.02.2018.

35 Nous avons relevé trois attestations de cyclo-touriste en fonction adjectivale (épreuve/club/randonnée cyclo-touriste).

36 Dans un article paru dans le journal Le Soir (14. 09. 2018), cependant, touristonautes se réfère à ceux qui aimeraient faire du tourisme dans l'espace (ou spatiotourisme). On peut donc parler de deux lexies homonymes. 
tourisme, équitourisme (tourisme à cheval), handi-tourisme, hélitourisme, locatourisme (sans doute par analogie avec locavorisme), m-tourisme (tourisme mobile, par smartphone), Poketourisme (tourisme lié à la chasse aux monstres virtuels de Pokémon Go), rando-tourisme $e^{37}$. Mais il y a également des lexies ainsi formées qui se réfèrent aux touristes eux-mêmes : agrotouriste ${ }^{38}$, cannatouristes, insta-touristes (des touristes qui ont un faible pour Instagram).

Toujours parmi les substantifs, nous avons pu repérer un seul mot-valise, qui comporte donc une syllabe commune entre deux lexies (cf. Sablayrolles 2019: 150) : il s'agit de toutourisme (toutou pour " chien » + tourisme), un tourisme de niche, au double sens du terme ( « segment de marché » et « abri pour les chiens »). D’autres cas semblent relever de l'amalgamation lexicale, bien qu'ils ne comportent qu'un seul phonème en commun et se rapprochent donc de la fractocomposition : aventouristes (jouant aussi sur la paronymie entre aventure et tourisme), spiritourisme (tourisme autour des spiritueux, par ex. le rhum), tourismatique (tourisme + informatique), volontourisme (tourisme à but humanitaire, qui associe vacances et bénévolat) et volon(-)touriste.

Au sein de la composition, il faut noter un hapax qui semble relever de la factorisation, à savoir d'une composition par amalgame où le segment commun n'est pas central ${ }^{39}$. Il s'agit de la lexie zapatouristes (zapat<istes $>$ touristes) : "Nous sommes beaucoup de zapatouristes étrangers et mexicains venus voir le dernier bastion intact de la guérilla révolutionnaire latino-américaine $»^{40}$.

D’après nos relevés, il n'y a qu'un seul cas de néologisme flexionnel, mais il ne s'agit vraisemblablement pas d'une création volontaire : attrape-touriste ${ }^{41}$ (au lieu de la forme lexicalisée attrape-touristes ${ }^{42}$.

La catégorie de la préfixation est assez fournie et comporte les lexies suivantes : anti(-) tourisme (pour laquelle il y a également quelques occurrences adjectivales), hyper(-)tourisme, micro(-)tourisme, nanotourisme ("Un ensemble de petites actions touristiques, que l'on peut monter autour de structures à imaginer $»^{43}$ ), post-tourisme, pro-tourisme, sous-tourisme, supertouristes ( A Attendus par un chauffeur à l'aéroport, hébergés à l'hôtel Le Meurice, au Bristol, au Plaza Athénée, au George V ou au Ritz, les cent supertouristes se sont vu proposer un programme de visite unique, extrêmement personnalisé $»^{44}$ ) et sur(-)tourisme. Pro-tourisme ne compte que deux occurrences, dont une correspond à un usage substantivé ( La concurrence et un débat couvent entre les 'pro-tourisme” et celles [les colonies de vacances] en quête de sens $»^{45}$ ) et l'autre a une fonction adjectivale

Pour cette dernière lexie, il peut s'agir tout aussi bien de composition régulière, car rando (pour randonnée) est une troncation tout à fait courante et n'est sans doute pas perçue comme un fractolexème.

38 Il s'agit à notre avis d'un fractocomposé dérivant de la troncation d'agrotourisme soudé avec touriste et non pas d'un composé agro- (au sens de " champ ») + touriste.

39 Cf. Sablayrolles (2019: 151).

40 www.courrierinternational.com, 05. 08. 2014.

${ }^{41}$ "Le hameau de Prévenchères, classé Plus beau village de France, s'avère loin d'être un attrape-touriste» (www.midilibre.fr, 30. 07. 2019).

42 Cf. www.larousse.fr, consulté le 28. 07. 2019.

43 www.courrier-picard.fr, 11. 05. 2017.

44 www.lefigaro.fr, 08. 12. 2016.

45 www.liberation.fr, 09. 07. 2018. 
(« L’office de tourisme vient aussi de lancer son nouveau site pro-tourisme particulièrement complet $\left.{ }^{46}\right)$.

En revanche, nous avons repéré seulement trois cas de suffixation : touristicité $^{47}$, touristification et son synonyme (hapax) : touristisation.

Les emprunts ${ }^{48}$ qui exploitent les bases ${ }^{\star}$ tourism $^{\star}$ et ${ }^{\star}$ tourist ${ }^{\star}$ ne sont pas très nombreux et il s'agit essentiellement de composés : dark tourists (des touristes qui aiment visiter les zones à risque), e-tourist visa, overtourism, slow(-)tourism, tourism-truck ("À la manière d'un food-truck, le "tourism-truck" accueillera les visiteurs sur les places de village, les parkings des stations balnéaires ») et voluntourism.

Homo touristicus est moins facilement analysable en fonction des matrices lexicogéniques. Il s'agit à notre avis d'un néologisme ludique qui détourne le modèle de l'homo œeconomicus popularisé par J.S. Mill et, en même temps, d'un allogénisme qui a recours à des formants latins. Dans notre corpus ${ }^{49}$ nous avons relevé onze attestations de cette lexie, parfois avec Homo en majuscule ${ }^{50}$ et traité comme un nom propre (avec l'emploi de l'article zéro) : "Les dispositifs contraignants se généralisent partout dans le monde, si bien qu'Homo touristicus n'est plus le bienvenu partout où il souhaite se rendre ${ }^{51}$, mais plus souvent en minuscule : «Très vite, le pathétique de l'homo touristicus, ersatz d'aventurier qui entend jouir du spectacle du monde sans rien céder de son confort, suscite la curiosité et la caricature $\aleph^{52}$. On peut aussi observer un début de série à partir d'homo touristicus : dans le corpus pointent des composés tels homo touristicus selfiensis (pour se moquer d'une nouvelle espèce humaine férue de selfies) et Homo touristicus vacancius, autant de néologismes (demi-)savants parodiques. Ces cas nous semblent très proches de ceux décrits par Sablayrolles, avec un « recours à la composition savante, non pour créer des termes dans des domaines spécialisés, mais bien au contraire, pour nommer des réalités quotidiennes, prosaïques » (Sablayrolles $2018: 88$ ).

Pour ce qui est des adjectifs, les matrices lexicogéniques attestées sont un peu moins nombreuses que pour les substantifs, ce qui peut s'expliquer statistiquement par le nombre inférieur de lexies adjectivales présentes dans le corpus. La composition « régulière " se confirme comme la catégorie la mieux représentée : artistico-touristique, commercialo-touristique, culturo-touristique, économico-touristique, folklo-touristique, historico-touristique, industrialo-touristique, ludo-touristique, médiatico-touristique, nolisé-touristique, politico-touristique, récréo(-)touristique et spiritualo-touristique, autant de lexies qui montrent, au niveau conceptuel, la profonde imbrication entre le tourisme et d'autres domaines et disciplines (arts, commerce, culture, économie, folklore, etc.).

Dans une autre série de composés, touristique prend plutôt la forme de l'allomorphe touristico-, en première position, et donne lieu à des lexies dont le statut néologique

46 www.leberry.fr, 28. 10. 2014.

47 "Le concept de touristicité, récemment développé, apparait, de ce point de vue très intéressant : il s'agit en effet de mesurer le potentiel touristique d'un territoire sur une échelle de 0 à 100 à travers quatre dimensions » (www.theconversation.com, 22. 07. 2018).

48 Nous avons exclu la lexie tourista (emprunt à l'espagnol, lexicalisé depuis longtemps), car elle n'est pas néologique.

49 On trouve aussi deux attestations (hors corpus) de homo touristicus dans Pronovost $(2012: 218,220)$.

50 C'est l'usage en botanique : genre avec majuscule, espèce en miniscule.

51 www.lemonde.fr, 12.03. 2020.

52 www.cqfd-journal.org, 02. 10. 2018. 
est plus douteux : touristico-comique, touristico-commercial, touristico-culturel, touristico-ferroviaire, touristico-gastronomique, touristico-gourmand, touristico-historique, touristico-humoristiques, touristico-immobilier, touristico-industriel, touristico-littéraires, touristico-religieux, touristico-scientifique, touristico-sportif et touristico-économique.

La composition hybride est à l'origine de neuf lexies, dont la plupart exploitent des formants déjà attestés pour les substantifs : astro-touristiques, cyclo(-)touristique, géo(-)touristique, mycotouristique, œno(-)touristique, pseudo-touristique, touristicide (il s'agit d'un hapax de l'écrivain Patrick Besson ${ }^{53}$ ) et le binôme polarisé tourismophile/tourismophobe.

La fractocomposition est également présente, grâce aux lexies agri/agro(-)touristique, biotouristique avec l'omniprésent formant bio- pour «biologique » (et non pas « relatif à la biologie ", ce qui en ferait une composition hybride), cannatouristique, éco(-)touristique et éco-agro-touristique.

Quant à la matrice de la préfixation, elle a fourni huit lexies : anti(-)touriste et anti(-) touristique, hyper(-)touristique, mi-touristique, para(-)touristique (dans les contextes, on évoque des structures/activités/produits para-touristiques, que l'on peut donc assimiler à ce qui relève à proprement parler du tourisme), post-touristique, semi-touristique et ultra(-)touristique.

La lexie vélotouristique, qui d'après sa configuration morphologique serait un composé (vélo + touristique), nous semble en revanche dériver par suffixation de vélotouriste.

Nous avons repéré seulement un adjectif emprunté : il s'agit de l'anglicisme touristy (équivalent de touristique, avec une connotation péjorative), hapax attesté dans un quotidien québécois :

Et que veulent voir ses clients, des touristes de tous âges, essentiellement canadiens-anglais, américains, français et montréalais (l'hiver), qui le sollicitent pour des visites privées? "Rien de touristy, mais ils veulent aller dans le Vieux-Montréal ou au marché Jean-Talon! Le "local" est populaire, bien manger aussi $»^{54}$.

Terminons notre passage en revue des procédés de formation par un mot-valise (qui est un autre hapax) : toutouristique : toutou + touristique, avec un redoublement syllabique déjà attesté dans toutourisme:

L'office du tourisme de Troyes souhaite faciliter la vie quotidienne des visiteurs qui découvrent la ville en compagnie de leur chien. Pour cela, un bar à toutou est disponible dans le hall. Mais ce n' est pas tout, chaque propriétaire se verra remettre un pack d'accueil contenant une écuelle, un sachet de friandises, un magazine 30 millions d'amis, un sac pour ramasser les déjections canines ainsi qu' un guide toutouristique de Troyes ${ }^{55}$.

\footnotetext{
www.lepoint.fr, 25. 07. 2015.

www.ledevoir.com, 15. 07. 2017.

www.lest-eclair.fr, 20. 08. 2016.
} 


\section{Variation diatopique et équivalents autochtones}

Certains néologismes se manifestent en priorité dans des contextes francophones spécifiques ou présentent des liens, plus ou moins évidents, avec des anglicismes. Tout d'abord, ciné(-)tourisme (14 occ.) est l'équivalent autochtone de deux anglicismes : set-jet et set-jetting, qui à leur tour réaménagent par permutation les formes jet-set et jet-setting: "Si le ciné-tourisme, à l'instar du tourisme de littérature, n'est pas né d'hier, producteurs et gouvernements s'associent de plus en plus pour faire du $7^{e}$ art une vitrine promotionnelle visant à dynamiser les visites $»^{56}$. La plupart des attestations de ciné(-)tourisme proviennent de sources francophones, majoritairement québécoises.

Sur la base des sources de presse, il est permis d'affirmer que récréotourisme (tourisme récréatif, 78 occ.) et son dérivé récréo(-)touristique, bien plus répandu (1500 occ., précédé par des noms tels " projet ", « développement », «station », « site ", etc.), sont deux québécismes. Un autre québécisme, bien qu'il s'agisse d'un hapax, est égotourisme, qui fait écho à égoportrait, équivalent autochtone de selfie $\mathrm{e}^{57}$.

Pour agro(-)tourisme (619 occ.) et agro(-)touriste (9 occ.), l'examen des contextes montre une très forte présence de la lexie au Canada. En revanche, la forme agri(-)tourisme (228 occ.) paraît circuler de manière plus homogène dans la sphère francophone, avec la France en première position, suivie de la Suisse. Au Canada on dénombre seulement 7 occurrences de cette lexie : c'est évidemment agro(-)tourisme qui s'est imposé.

Chasse-touristes est un autre hapax, attesté au Niger et déjà cité plus haut, qui renvoie à une pratique observable en particulier dans le contexte africain. Pescatourisme ( $55 \mathrm{occ}$.), néologisme qui désigne la pêche touristique, est présent de manière prioritaire dans des sources algériennes et tunisiennes. Toujours en Tunisie, on trouve deux occurrences sur les trois totales de tourisme-charia, une pratique préconisée, semble-t-il, par le " conseil des imams $»^{58}$.

Pour passer aux rapports entre lexies autochtones et allogènes, sur(-)tourisme est l'équivalent, sémantique et "calqué " (emploi du même procédé morphologique), de l'anglicisme overtourism ( 6 occ.), qui indique un excès de touristes dans un lieu ${ }^{59}$, et de la forme hybride overtourisme (8 occ.). Avec ses 311 occurrences, sur(-)tourisme semble déjà bien implanté, beaucoup plus que son synonyme hyper(-)tourisme, qui ne compte pour l'instant que 5 attestations.

Volontourisme, en revanche, paraît une création sous influence de l'anglais volontourism, car le sème "bénévole » dans le fractolexème volont- n'est pas tout de suite transparent.

La lexie $m$-tourisme (47 occ.) comporte un fractolexème $m$-valant pour mobile, ou plutôt pour la forme homonyme anglaise " mobile »; dans cette deuxième hypothèse, étayée par la syntaxe anglo-saxonne du composé (déterminé/déterminant), on peut donc encore parler d'une création sous influence.

www.latribune.fr, 25. 05. 2015.

Cf. Vincent (2020).

www.tunisienumerique.com, 07. 04. 2017.

https://fr.wiktionary.org/wiki/surtourisme. 


\section{Conclusions}

Nous sommes loin, bien entendu, d'avoir fait le tour de la néologie du tourisme, un domaine extrêmement dynamique et qui sera appelé à se renouveler ultérieurement à la suite de la pandémie de Covid-19. Cependant, cette première étude ${ }^{60}$, qui s'est focalisée sur les bases ${ }^{\star}$ tourism ${ }^{*}$ et ${ }^{\star}$ tourist ${ }^{\star}$, a permis d'apprécier le foisonnement néologique de ce secteur et d'évaluer les principaux mécanismes de formation. La productivité des deux bases examinées est remarquable, d'un point de vue quantitatif mais aussi pour ce qui est de la variété des matrices lexicogéniques qui s’y greffent.

Les lexies de la série analysée montrent une forte prédilection pour la composition, dans tous ses avatars (en ordre de fréquence : régulière, hybride, par fractocomposition et mot-valisation), ce qui ne va pas dans le même sens des tendances générales du français contemporain identifiées par Cartier (2019), où la composition vient en deuxième place (à l'origine de seulement 7,32 \% des néologismes) et bien après la préfixation ( $75,87 \%$ des formes). Comment interpréter une préférence si marquée pour la composition dans la série ${ }^{\star}$ tourism $^{*} /{ }^{*}$ tourist $^{\star}$ ? Ce procédé permet de souder deux éléments et fournit une dénomination synthétique pour des pratiques plus ou moins nouvelles. On se rapproche ainsi de la terminologie, où la composition sert à créer une pléthore de catégories et sous-catégories. La brièveté et l'efficacité d'une formule condensée peuvent jouer un rôle dans le foisonnement de cette matrice néologène, très exploitée par les journalistes, qui relaient souvent des innovations créées par d'autres locuteurs, dans ce cas spécifique les personnes travaillant dans le marketing touristique.

Par rapport à d'autres domaines, les emprunts et les créations sous influence paraissent peu nombreux. On peut formuler l'hypothèse que le tourisme francophone, même dans ses innovations, puise à un patrimoine naturel et culturel autochtone qui rend superflu le recours à la matrice externe.

En perspective, il serait stimulant de poursuivre l'exploration du vocabulaire touristique par une étude comparative (quantitative et qualitative), avec d'autres langues européennes. Les questions suivantes se posent : combien de lexies ont été générées dans chaque langue à partir de la base tour-? Quelle est l'influence de l'anglais dans les langues prises en examen? Quel est le taux de recouvrement entre les différentes langues? Mais il ne s'agit que de questions préliminaires, d'autres seront suggérées par les faits linguistiques eux-mêmes.

\section{BIBLIOGRAPHIE}

Angelillo, M. (2015) : «Da incredibile a credibile : strategie nazionali di promozione turistica in India », Lingue Culture Mediazioni, 2, 1 https://www.ledonline.it/index.php/LCM-Journal/article/download/798/701, consulté le 25. 06. 2020.

${ }_{60}$ Nous poursuivons l'étude de la néologie et de la terminologie du tourisme au sein du projet sur les Humanités Numériques qui est en cours au Département des Langues et Littératures Etrangères de l'Université de Vérone (http://dh.dlls.univr.it). 
Aquilina, M., Mahéo, C. et Pugnière-Saavedra, F., «Introduction générale ». In Aquilina, M., Mahéo, C. et Pugnière-Saavedra, F. (2018) : La communication touristique : vers de nouvelles interfaces? Du discours aux représentations territoriales, Québec : Presses de l'Université Laval, pp. 1-13.

Baider, F., Burger, M. et Goutsos D. (2005) : La communication touristique. Approches discursives de l'identité et de l'altérité. Tourist communication. Discursive Approaches to Identity and Otherness, Paris : L'Harmattan.

Cartier, E. (2019) : " Néoveille, plateforme de repérage et de suivi des néologismes en corpus dynamique ", Neologica, 13, pp. 23-54.

Christin, R. (2014) : L'usure du monde. Critique de la déraison touristique. Montreuil : Éditions L'échappée.

Decroly, J.-M. et al. (2006) : Tourisme et société. Mutations, enjeux et défis. Bruxelles : Éditions de l'Université de Bruxelles.

Gotti, M., Naci S. et Sala, M. (2017) : Way of seeing, ways of being. Representing the voices of tourism. Bern : Peter Lang.

Jacquet-Pfau, C. : « Statut et productivité de quelques éléments de formation hyperbolique : archi, hyper, méga, super, ultra... à travers un corpus de presse ». In Tallarico G., Humbley J. et Jacquet-Pfau C. (2020) : Nouveaux horizons pour la néologie en français. Hommage à Jean-François Sablayrolles, Limoges : Lambert-Lucas, pp. 135-151.

Kalfon, P. (2009) : Tourisme et innovation. Paris, L'Harmattan.

Kortas, J. (2009) : « Les hybrides lexicaux en français contemporain : délimitation du concept », Meta, 54/3, pp. 533-550.

Margarito, M. (2000) : L'Italie en stéréotypes. Analyse de textes touristiques. Paris : L'Harmattan.

Nigro, M. G. (2006) : Il linguaggio specialistico del turismo. Aspetti storici, teorici e traduttivi. Rome : Aracne. Paquot, T. : "Pour une épistémologie du tourisme? ». In Morisset, L.K., Sarrasin, B. et Éthier, B. (2012) : Épistémologie des études touristiques. Montréal : Presses de l'Université du Québec, pp. 9-23.

Paquot, T. (2014) : Le voyage contre le tourisme. Paris : Association culturelle Eterotopia France.

Piccato, M. (2014) : Création et exploitation d'un corpus trilingue du tourisme (français/italien/anglais) en vue de la réalisation d'une base de données lexicale informatisée. Paris : Hermann.

Piccato, M. (2015) : Glossaire trilingue du tourisme (français/italien/anglais). Une étude terminologique comparée. Paris : Hermann.

Picone, M. (1996) : Anglicisms, Neologisms and Dynamic French. Amsterdam : John Benjamins.

Pronovost, G. : « Regard conclusif. La construction de la notion de "touriste" dans les sciences sociales ». In Morisset, L.K., Sarrasin, B. et Éthier, B. (2012) : Épistémologie des études touristiques. Montréal : Presses de l'Université du Québec, pp. 217-226.

Renner, V. : « Réflexions sur l'usage et le traitement lexicographique des emprunts à l'anglais et des officialismes dans la langue française du tourisme ». In Ligas, P. et Frassi, P. (2012) : Lexiques, Identités, Cultures. Vérone : QuiEdit, pp. 243-253.

Sablayrolles, J.-F. : " Des innovations lexicales ludiques dans des situations d'énonciation marginales ou spécifiques ». In Winter-Froemel, E. et Demeulanaere, A. (2018) : Jeux de mots, textes et contextes. Berlin : De Gruyter, pp. 77-94.

Sablayrolles, J-F. (2019) : Comprendre la néologie. Conceptions, analyses, emplois. Limoges : Lambert-Lucas, coll. « La Lexicothèque ».

Tallarico, G. et Humbley, J. (2020) : «Quelle néologie pour l'œnotourisme? Stratégies françaises et italiennes en confrontation », Neologica, 14, pp. 203-223.

Vincent, N. : «Quand l'actualité impose des néologismes, les dictionnaires professionnels peuvent-ils rivaliser avec les dictionnaires collaboratifs ? ». In Tallarico, G., Humbley, J. et Jacquet-Pfau, C. (2020) : Nouveaux horizons pour la néologie en français. Hommage à Jean-François Sablayrolles. Limoges : Lambert-Lucas, pp. 69-85.

\section{Giovanni Tallarico}

Département des Langues et Littératures Étrangères

Université de Vérone

41, Lungadige Porta Vittoria

37129 Vérone (Italie)

giovanni.tallarico@univr.it 